\title{
REAÇÃO DA CÂNFORA COM BOROIDRETO DE SÓDIO: UMA ESTRATÉGIA PARA O ESTUDO DA ESTEREOQUÍMICA DA REAÇÃO DE REDUÇÃ̃O
}

\author{
Péricles B. Alves* \\ Departamento de Química, Centro de Ciências Exatas e Tecnológicas, Universidade Federal de Sergipe, Cidade Universitária \\ "Prof. José Aloísio de Campos", Av. Mal. Rondon, s/n, 49100-000 São Cristóvão - SE, Brasil \\ Mauricio M. Victor \\ Departamento de Química Orgânica, Instituto de Química, Universidade Federal da Bahia, Campus de Ondina, 40170-290 \\ Salvador - BA, Brasil \\ Recebido em 6/1/10; aceito em 25/8/10; publicado na web em 25/10/10

\begin{abstract}
REACTION OF CAMPHOR WITH SODIUM BOROHYDRIDE: A STRATEGY TO INTRODUCE THE STEREOCHEMICAL ISSUES OF A REDUCTION REACTION. Reduction of camphor to a mixture of borneol and isoborneol was performed using $\mathrm{NaBH}_{4}$ as the reducing agent under suitable conditions. Although more effective reduction was accomplished using toxic methanol, an alternative non-toxic ethanolic system is described. This experiment is important to introduce undergraduate students in reductive procedures, and can be used to show details on stereoselective procedures on carbonyl moieties (facial diastereoselectivity, BürgiDunitz trajectory, diastereomeric excess).
\end{abstract}

Keywords: camphor; reduction; sodium borohydride.

\section{INTRODUÇÃO}

Coube a Julius Bredt (1855-1938), químico alemão nascido em Berlim, propor a estrutura da cânfora (1,7,7-trimetilbiciclo-[2.2.1]heptan-2-ona) como conhecida hoje: um monoterpeno bicíclico cetônico, de fórmula molecular $\mathrm{C}_{10} \mathrm{H}_{16} \mathrm{O}$, com esqueleto carbônico do tipo norbornano. ${ }^{1} \mathrm{~A}$ cânfora pode ser obtida do canforeiro (Cinnamomum canphora (L.) J. Presl), da família Lauraceae, nativa do Taiwan, Japão e China. Outras fontes naturais são as plantas Dryobalanops aromatica e Ocotea usambarensis. Industrialmente a cânfora é obtida a partir do $\alpha$-pineno, ${ }^{2}$ composto natural abundante presente no óleo da terebentina. Suas características organolépticas são odor forte e penetrante, sabor amargo e levemente fria ao tato. Suas propriedades biológicas são bastante variadas, indo desde o uso como excitante em casos de paradas cardíacas resultantes de febres infecciosas até seus conhecidos usos contra gripes, resfriados e complicações inflamatórias. ${ }^{3}$ É também utilizada contra histeria, nervosismo, neuralgia e diarreia por uso interno, e externamente empregada contra reumatismos, bronquites e inflamações. É muito utilizada na indústria química para obtenção de plásticos e de nitrocelulose; como base de filmes fotográficos, repelente de traças, preservativo em cosméticos e na formulação de medicamentos. ${ }^{4}$

Sua estrutura foi determinada através de reações químicas, num trabalho laborioso devido às dificuldades da época e ao desconhecimento de algumas reações que conhecemos hoje. A contribuição de Julius Bredt deve-se não somente à elucidação da estrutura da cânfora, mas também a sua estereoquímica e produtos de reações.

A introdução de um átomo de hidrogênio nucleofílico (hidreto $\mathrm{H}^{-}$) a uma carbonila é uma reação de extrema importância em Química Orgânica. ${ }^{5}$ Vários reagentes redutores (fontes de hidreto) estão disponíveis comercialmente, destacando-se entre eles o hidreto de lítio e alumínio $\left(\mathrm{LiAlH}_{4}\right)$ e o boroidreto de sódio $\left(\mathrm{NaBH}_{4}\right)$. A redução de aldeídos e cetonas com estes reagentes fornece alcoóis primários e secundários,

*e-mail: pericles@ufs.br

"Dedicado ao Prof. Hans Vietler pelos seus 70 anos e a sua importante

contribuição à Química Brasileira. respectivamente, em altos rendimentos e com fácil purificação. No entanto, devido ao seu custo moderado em relação ao $\mathrm{LiAlH}_{4}$ e à facilidade de manipulação, o $\mathrm{NaBH}_{4}$ é preferencialmente empregado.

Neste trabalho propõe-se o emprego de uma reação simples de redução da cânfora empregando-se o boroidreto de sódio, ${ }^{6}$ facilmente executável em aulas de graduação (Figura 1). O produto da redução, uma mistura de isoborneol e borneol, foi caracterizado através de técnicas corriqueiramente disponíveis (cromatografia gasosa e infravermelho).

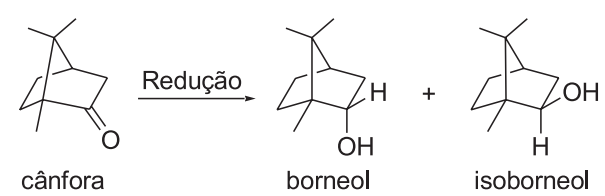

Figura 1. Compostos formados na redução da cânfora

A redução da cânfora é um bom modelo para abordar assuntos como elucidação estrutural, aspectos estereoquímicos e caracterização dos produtos de reação. A baixa toxidade da cânfora (DL50 em ratos: 3000 $\mathrm{mg} / \mathrm{kg})^{7}$ permite que os alunos a manipulem sem maiores cuidados em aulas práticas, e seu preço bastante acessível ${ }^{8}$ é mais um fator a favorecer a utilização deste experimento. Além destes fatores, publicações recentes tratando da análise do espectro de RMN da cânfora, ${ }^{9}$ bem como de sua oxidação empregando métodos alternativos e ambientalmente corretos, ${ }^{10}$ permitem a criação de experimentos conjugados de compreensão dos fenômenos de oxidação e redução, bem como de análise espectral, levando à minimização de perdas e incutindo nos alunos a preocupação sobre reciclagem dos produtos obtidos em sua instrução.

\section{PARTE EXPERIMENTAL}

\section{Redução com $\mathrm{NaBH}_{4}$ em metanol}

Em um frasco erlenmeyer de $100 \mathrm{~mL}$ adicionou-se $1,0 \mathrm{~g}(6,57$ mmoles) de cânfora e $10 \mathrm{~mL}$ de metanol. Em seguida, foi adicionado $1,0 \mathrm{~g}$ (26,43 mmoles) de boroidreto de sódio à solução, em pequenas porções, até completa dissolução, mantendo-se sempre o sistema sob 
agitação manual. Feito isto, o erlenmeyer foi aquecido sobre chapa de aquecimento em capela de exaustão até entrar em refluxo (visualizado nas paredes internas do frasco). Manteve-se um leve aquecimento durante 5 min (pode-se buscar um ponto menos quente na chapa de aquecimento, normalmente na borda, ou retirá-lo a intervalos do contato com a superfície aquecida), de forma a reduzir ao mínimo a perda por volatilização, agitando-se manualmente o frasco de forma ocasional. Passado este tempo aguardou-se o resfriamento e adicionaram-se cerca de $30 \mathrm{~mL}$ de uma mistura água-gelo. A solução tornou-se imediatamente esbranquiçada pela precipitação de um sólido, que foi filtrado em funil de Büchner, permitindo-se a secagem por sucção de ar por cerca de $5 \mathrm{~min}$. O sólido foi transferido para um frasco erlenmeyer de $100 \mathrm{~mL}$, dissolvido em $30 \mathrm{~mL}$ de éter etílico ${ }^{11}$ e seco pela adição de sulfato de sódio anidro. A filtração para um frasco previamente tarado e evaporação do solvente em capela de exaustão sob leve aquecimento levou à obtenção do bruto reacional. Rendimento aproximado de $85 \%$.

\section{Redução com $\mathrm{NaBH}_{4}$ em etanol}

Em um balão de $100 \mathrm{~mL}$ adicionou-se 1,0 g (6,57 mmoles) de cânfora e $10 \mathrm{~mL}$ de etanol absoluto. Após a dissolução foi adicionado $1,0 \mathrm{~g}$ (26,43 mmoles) de boroidreto de sódio à solução, em pequenas porções, mantendo-se sempre a suspensão sob agitação manual. O balão foi preso a uma haste com um agarrador, um condensador de refluxo foi conectado ao balão e o sistema foi aquecido a refluxo por 15 min com auxílio de uma manta de aquecimento. Passado este tempo aguardou-se o resfriamento e adicionaram-se $15 \mathrm{~mL}$ de uma mistura água-gelo. A solução tornou-se imediatamente esbranquiçada pela precipitação de um sólido, que foi filtrado em funil de Büchner, permitindo-se a secagem por sucção de ar por cerca de $5 \mathrm{~min}$. O sólido foi transferido para um frasco erlenmeyer de $100 \mathrm{~mL}$, dissolvido em $30 \mathrm{~mL}$ de éter etílico ${ }^{11}$ e seco pela adição de sulfato de sódio anidro. A filtração para um frasco previamente tarado e evaporação do solvente em capela de exaustão sob leve aquecimento levou à obtenção do bruto reacional. Rendimento aproximado de $70 \%$.

\section{INSTRUMENTAÇÃO E MODO DE OPERAÇÃO NA CARACTERIZAÇÃO DOS PRODUTOS DE REDUÇÃO DA CÂNFORA}

\section{Espectroscopia no infravermelho (IV)}

O espectro vibracional na região do infravermelho foi registrado em espectrofotômetro FT-IR Bomem MB 100, em pastilha de $\mathrm{KBr}$ confeccionada com 3-5 mg de amostra e $100 \mathrm{mg}$ de $\mathrm{KBr}$. Os valores para a absorção foram referidos em número de onda, e para a calibração do equipamento foi utilizada a banda padrão de transmitância de filme de poliestireno a $1601 \mathrm{~cm}^{-1}$.

\section{Cromatografia gasosa com detector de ionização de chamas (CG-DIC)}

As análises quantitativas da mistura reacional foram realizadas em um cromatógrafo gasoso equipado com detector de ionização de chamas (DIC) Shimadzu GC-17A, sob as seguintes condições operacionais: coluna capilar de sílica fundida ZB-5MS (5\% dimethylpolysiloxane) com $30 \mathrm{~m}$ x $0.25 \mathrm{~mm}$ i.d. x $0.25 \mu \mathrm{m}$ de filme, usando He como gás de arraste com fluxo de $1,2 \mathrm{~mL} / \mathrm{min}$. A temperatura foi mantida a 50 ${ }^{\circ} \mathrm{C}$ por $2 \mathrm{~min}$, depois aumentada de $4{ }^{\circ} \mathrm{C} / \mathrm{min}$ até atingir $200{ }^{\circ} \mathrm{C}$. Em seguida, aumentada de $15^{\circ} \mathrm{C} / \mathrm{min}$ até atingir $300^{\circ} \mathrm{C}$, mantendo-se esta temperatura constante por $15 \mathrm{~min}$; a temperatura do injetor foi $250^{\circ} \mathrm{C}$ e a temperatura do detector (ou interface) de $280{ }^{\circ} \mathrm{C}$. Foi injetado um volume de $0,5 \mu \mathrm{L}$ da mistura reacional dissolvida em acetato de etila.

\section{Cromatografia gasosa acoplada à espectrometria de massas} (CG-EM)

A análise qualitativa da mistura reacional foi realizada em cromatógrafo a gás acoplado a espectrômetro de massa CG-EM (Shimadzu, modelo QP 5050A), equipado com coluna capilar de sílica fundida J\&W Scientific (5\%-phenyl-95\%-dimethylpolysiloxane) de $30 \mathrm{~m}$ x $0.25 \mathrm{~mm}$ i.d., $0.25 \mu \mathrm{m}$ de filme, usando He como gás de arraste com fluxo de 1,2 $\mathrm{mL} / \mathrm{min}$. As condições cromatográficas da análise foram as mesmas utilizadas para CG-DIC. As condições do EM foram detector de captura iônica operando por impacto eletrônico e energia de impacto de $70 \mathrm{eV}$; velocidade de varredura 1.000; intervalo de varredura de 0,50 fragmentos/s e fragmentos detectados na faixa de 40 a $500 \mathrm{Da}$.

\section{Cálculo do índice de retenção (IR) - ferramenta auxiliar na identificação dos produtos da redução da cânfora}

Observando-se os espectros de massas do isoborneol e do borneol (material suplementar, Figura 1S) nota-se grande semelhança no padrão de fragmentação entre ambos, o que dificulta uma identificação inequívoca da mistura dos dois compostos, apesar da técnica CG-EM ser uma poderosa ferramenta de análise e identificação.

Uma maneira de superar esta dificuldade foi a utilização do índice de retenção (IR), que é valor numérico obtido pela interpolação, relacionando o tempo de retenção do composto em estudo com o tempo de retenção de uma série homóloga de mistura de hidrocarbonetos lineares. Os IR para o isoborneol (1159) e borneol (1168) foram calculados através de uma série homóloga de hidrocarbonetos lineares (faixa de nC9-nC18), aplicando a Equação de van den Dool e Kratz: ${ }^{12}$

$$
\mathrm{IR}=100 \mathrm{i}\left[\frac{\operatorname{Tr}(\mathrm{X})-\operatorname{Tr}(\mathrm{HA})}{\operatorname{Tr}(\mathrm{HD})-\operatorname{Tr}(\mathrm{HA})}\right]+100 \mathrm{~N}
$$

onde: $\mathrm{IR}=$ índice de retenção; $\operatorname{Tr}(\mathrm{X})=$ tempo de retenção do composto problema; $\operatorname{Tr}(\mathrm{HA})=$ tempo de retenção do hidrocarboneto que elui antes do composto $(\mathrm{x})$; $\operatorname{Tr}(\mathrm{HD})=$ tempo de retenção do hidrocarboneto que elui depois do composto $(\mathrm{x})$; $\mathrm{i}=$ diferença do número de carbonos entre os hidrocarbonetos que eluem depois e antes; $\mathrm{N}=$ número de carbonos do hidrocarboneto que elui antes do composto (x).

Os espectros de massas dos constituintes da mistura reacional foram comparados com espectros de massas do banco de dados (NIST21 e NIST107) do equipamento, bem como seus valores de IR com os descritos na literatura. ${ }^{13}$

\section{A REDUÇÃO DA CÂNFORA}

As reações de redução utilizando reagentes doadores de hidreto a carbonila mais disponíveis no mercado são as realizadas com boroidreto de sódio $\left(\mathrm{NaBH}_{4}\right)$ e hidreto de lítio e alumínio $\left(\mathrm{LiAlH}_{4}\right)$. A redução com boroidreto de sódio, além de menos onerosa, é um procedimento de manipulação mais fácil quando comparado à redução com hidreto de lítio e alumínio. A reatividade do $\mathrm{LiAlH}_{4}$ é bem superior à do $\mathrm{NaBH}_{4}$, devido a maior diferença de eletronegatividade $\left(\Delta_{\mathrm{X}}\right)$ da ligação Al-H $\left(\Delta_{\mathrm{X}}=0,5\right)$ em relação à ligação $\mathrm{B}-\mathrm{H}\left(\Delta_{\mathrm{X}}=0,1\right)$. No entanto, esta menor reatividade do boroidreto de sódio passa a ser uma vantagem, pois assim se transforma em um redutor mais seletivo. Aldeídos e cetonas são prontamente reduzidos pelo boroidreto de sódio, enquanto que ésteres, amidas, cloretos de ácido, anidridos e ácidos carboxílicos normalmente não são afetados ou requerem longos tempos de redução. 
Apesar do boroidreto de sódio ser largamente empregado em laboratórios e centros de instrução, seu mecanismo ainda é controverso. Wigfield e colaboradores ${ }^{14}$ propuseram vários estados de transição considerando apenas o ânion boroidreto $\mathrm{BH}_{4}^{-}$, menosprezando o papel do cátion sódio $\mathrm{Na}^{+}$. No entanto, esta teoria foi refutada por resultados experimentais que mostraram a importância da participação do cátion. ${ }^{15}$ Esta importância é reforçada por artigo recente, ${ }^{16}$ que mostra o papel coordenante do sódio em cálculos de estado de transição. Deste modo, o mecanismo mais aceito para a redução com boroidreto de sódio é aquele que envolve a complexação do oxigênio carbonílico com o $\mathrm{Na}^{+}$(semelhante a um ácido de Lewis), tornando o carbono carbonílico mais deficiente de elétrons (mais eletrofílico), facilitando a transferência do hidreto (Figura 2). O alcóxido formado nesta etapa de adição de hidreto transforma-se no mono-alcoxiboroidreto, ainda um agente redutor, que pode reduzir outras moléculas de carbonilado. O tetra-alcoxiboroidreto é hidrolisado pelo próprio solvente prótico (normalmente álcoois) ou, então, pela adição de água ou cloreto de amônio (menos comum), liberando o álcool.
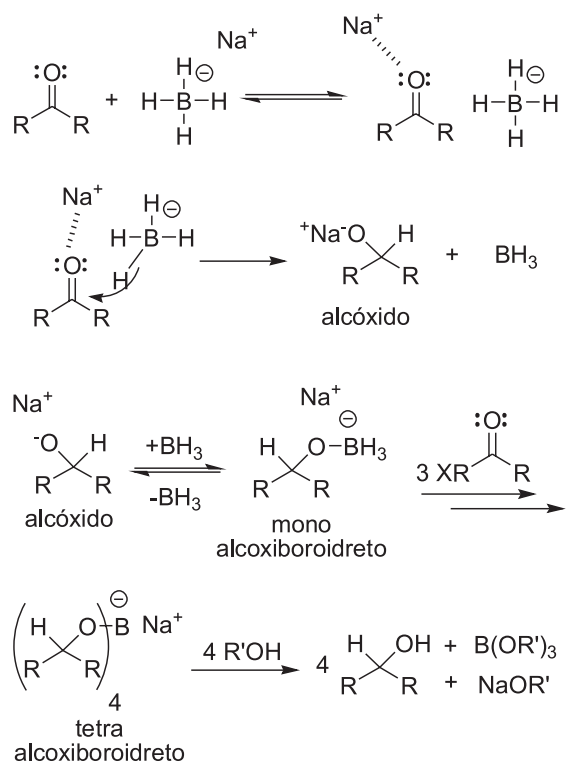

Figura 2. Mecanismo proposto para redução de carbonilados com $\mathrm{NaBH}_{4}$. Adaptado da ref. 5

Apesar da aceitação comum do papel do cátion no aumento da eletrofilicidade do carbono carbonílico, conforme descrito na etapa da complexação, pode-se verificar através de uma consulta rápida aos livros textos de Química Orgânica mais comumente empregados, ${ }^{17}$ que a maioria ${ }^{18}$ não adota o mecanismo como descrito acima, mesmo que de forma simplificada. Os textos didáticos remetem ao modelo de mecanismo descrito por Wigfield, onde apenas o ânion boroidreto participa da etapa de redução (Figura 3). Os livros textos mais avançados ${ }^{5,19}$ mostram o papel do cátion na redução da carbonila e, mesmo assim, não há uma forma consensual de representar esta simples transformação. ${ }^{20}$

A estereoquímica proveniente da redução da cânfora com boroidreto de sódio é muito interessante, principalmente devido a questões de reatividade da carbonila e a questões estruturais do sistema norbonano. Do ponto de vista reacional, é sabido que a aproximação do nucleófilo (o hidreto $\mathrm{H}^{-}$) ao carbono eletrofílico da carbonila é governada por interações dos orbitais de fronteira de cada uma das espécies envolvidas. Segundo esta abordagem, os elétrons no nucleófilo estão alocados no orbital ocupado de mais alta energia (HOMO) e a interação deste par eletrônico dar-se-á com o orbital desocupado de mais baixa energia (LUMO) presente no composto
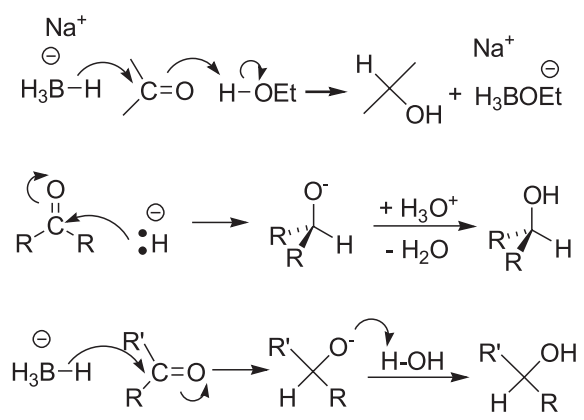

Figura 3. Exemplos de mecanismos encontrados nos livros textos de $Q O$

carbonilado. O LUMO do composto carbonilado é descrito como o orbital antiligante do tipo $\pi^{*}$, obtido a partir da combinação fora de fase entre os orbitais atômicos ' $p$ ' do carbono e do oxigênio. Neste orbital, o coeficiente é maior no átomo de carbono, que representa o sítio eletrofílico. Assim, durante a aproximação do nucleófilo, o mesmo irá se direcionar ao átomo de carbono, e com esta trajetória o nucleófilo rico em elétrons evitará a repulsão eletrônica desestabilizadora com a região rica em elétrons, representada pelo orbital $\pi$ ao redor do átomo de oxigênio. No entanto, este ângulo de aproximação, que durante muito tempo se pensou perpendicular $\left(90^{\circ}\right)$ ao plano dos átomos da carbonila, é na verdade ao redor de $107^{\circ}$, e esta trajetória recebeu o nome de seus descobridores, H. B. Bürgi e J. D. Dunitz. ${ }^{21}$ A trajetória de Bürgi-Dunitz (Figura 4), como ficou conhecida, foi determinada através da análise de dados cristalográficos de 6 moléculas que continham em suas estruturas tanto uma carbonila como uma espécie nucleofílica, e como estas estruturas se arranjavam no espaço. Montando um gráfico das distâncias Nü---C=O e sua relação com o plano RR' $\mathrm{C}=\mathrm{O}$ os autores, num magnífico trabalho, mostraram que os nucleófilos estão alinhados sobre um vetor que tem um ângulo de $107^{\circ}$ com respeito ao plano da carbonila. Trabalhos posteriores mostraram ser esta, também, a trajetória que um nucleófilo busca a fim de maximizar a superposição do HOMO do nucleófilo com o LUMO da ligação $\mathrm{C}=\mathrm{O}$ e minimizar a repulsão com a densidade eletrônica localizada no oxigênio (no orbital $\pi$ de natureza ligante).

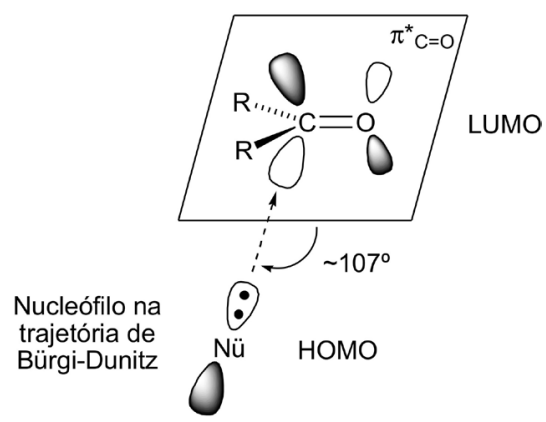

Figura 4. Trajetória de Bürgi-Dunitz

Tendo-se definido como o hidreto nucleofílico se aproxima da carbonila, pode-se aplicar este modelo sobre a estrutura da cânfora (sistema 1,7,7-trimetilbiciclo[2.2.1]heptan-2-ona). Esta aproximação poderia acontecer por ambas as faces da carbonila da cânfora, mas não com igual probabilidade (Figura 5). Pode-se facilmente verificar com o uso de modelos moleculares que a aproximação é mais facilitada pela face inferior (aproximação do tipo endo), a qual leva à formação do álcool com a hidroxila na posição pseudoequatorial, ou seja, o álcool exo, neste caso chamado de isoborneol. Se o ataque do hidreto ocorre pela face superior (ataque exo), uma grande repulsão estérica é criada pela proximidade de uma das metilas geminais na trajetória de Bürgi-Dunitz de aproximação à carbonila. Assim, uma 
maior energia do estado de transição será alcançada, e a formação do álcool com a hidroxila na posição pseudoaxial (álcool endo, borneol) ocorrerá em menor proporção.

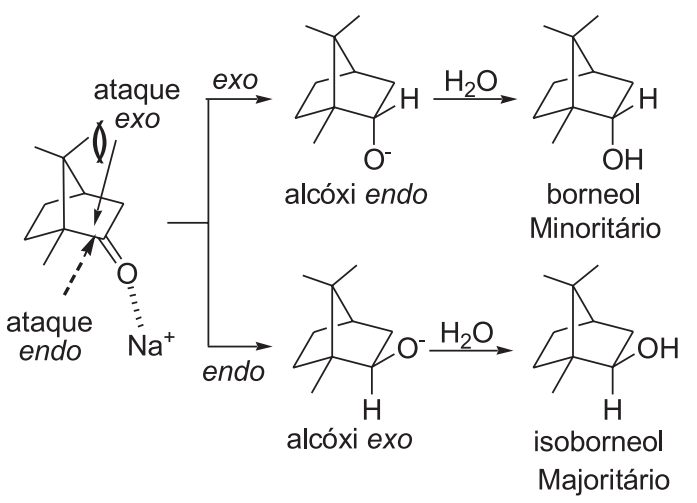

Figura 5. Estereoquímica do ataque do boroidreto de sódio à cânfora

No nosso caso, a determinação da proporção da mistura isomérica obtida (borneol mais isoborneol) e do isômero majoritário foi efetuada com o uso de cromatografia gasosa (material suplementar, Figura $2 S)$. Um padrão de (-)-borneol comercial ${ }^{22}$ foi previamente injetado no CG-DIC, bem como um padrão de cânfora racêmica, a fim de se poder determinar a total conversão na redução. Este procedimento de verificação de total conversão se mostrou mais importante no caso de utilização de etanol como solvente, uma vez que a redução se tornou mais lenta. O mesmo acompanhamento de total conversão foi feito através da realização de espectros de infravermelho (material suplementar, Figuras 3S e 4S) em pastilhas de $\mathrm{KBr}$, observando-se principalmente o desaparecimento do sinal referente à carbonila $\left(\sim 1743 \mathrm{~cm}^{-1}\right)$ e o aparecimento do sinal correspondente à hidroxila (sinal largo entre 3500 e $3300 \mathrm{~cm}^{-1}$ ). A análise de CG-EM também foi realizada nas misturas de borneol e isoborneol. ${ }^{23}$

Por fim, na disciplina Síntese e Caracterização de Compostos Orgânicos do curso de Química Tecnológica, foi realizado um projeto de busca de metodologia de redução da cânfora empregando-se etanol como substituto do metanol, de forma a permitir a realização de experimentos com solventes menos tóxicos. Neste projeto ficou clara a necessidade de um sistema mais adaptado para execução do refluxo com etanol (Parte Experimental), pois a reação se tornou mais lenta. ${ }^{24}$ Embora o rendimento tenha sido um pouco inferior ( $70 \%$ com etanol contra cerca de $85 \%$ com metanol), a possibilidade de uso do etanol mostrou-se bastante satisfatória do ponto de vista de diminuição de riscos à saúde no laboratório, devido a menor toxicidade do solvente. Outro dado interessante foi a redução da diastereosseletividade da redução. Enquanto a reação com boroidreto de sódio em metanol mostrou uma proporção de cerca de 6:1 (isoborneol:borneol), a redução em etanol teve seletividade mais baixa $(3: 1){ }^{25}$

Deste modo, em conjugação com um experimento de oxidação da cânfora, ${ }^{10}$ é possível realizar um trabalho de laboratório onde vários assuntos podem ser abordados (necessidade de reciclagem de produtos de laboratório e/ou uso em experimentos subsequentes; conceitos de oxidação e redução; estereoquímica e conceitos correlatos com diastereosseletividade, excesso diastereoisomérico e controle estérico; discussão de mecanismos e influência de solventes; conceitos de orbitais; análises espectroscópicas), enriquecendo a formação de nossos graduandos na área de Química Orgânica Experimental.

\section{CONCLUSÕES}

O experimento de redução da cânfora foi realizado em escala semimicro, com o emprego de uma metodologia clássica de redução através de hidreto de um composto carbonílico e de reagentes comercialmente disponíveis. Por se tratar de uma reação rápida, pode ser realizada em uma aula de $2 \mathrm{~h}$.

A redução da cânfora foi satisfatória, com excelente grau de pureza e bons rendimentos, podendo ser também realizada com solvente pouco tóxico (etanol), o que permite sua execução até em laboratórios desprovidos de exaustão. A abordagem proposta neste experimento envolveu aspectos de Síntese Orgânica, análise espectroscópica, estereoquímica e mecanismo de reação, permitindo aos estudantes a discussão e aprofundamento de aspectos teóricos destes conhecimentos através do curso experimental.

\section{MATERIAL SUPLEMENTAR}

O material suplementar contém os espectros de massas do borneol e isoborneol, o cromatograma dos produtos da redução da cânfora e os espectros de infravermelho, disponíveis em http://quimicanova. sbq.org.br, na forma de arquivo PDF, com acesso livre.

\section{AGRADECIMENTOS}

Ao CNPq, aos alunos da disciplina Síntese e Caracterização de Compostos Orgânicos por seu empenho e dedicação, e aos discentes A. Aquino, H. C. R. de Jesus, G. B. Barin, D. A. Rodrigues, A. S. Costa, E. dos S. Niculau, G. R. S. Andrade e R. L. de Farias pela realização dos experimentos, cromatogramas e obtenção dos fragmentogramas. Os espectros de infravermelho requerem nosso agradecimento à Profa. Z. N. Rocha (UFBA).

\section{REFERÊNCIAS}

1. Bredt, J.; Chem. Ber. 1893, 26, 3047.

2. Ritter, J. J.; J. Am. Chem. Soc. 1933, 55, 3322.

3. http://botanical.com/botanical/mgmh/c/campho13.html, acessada em Setembro 2009.

4. Enciclopédia Barsa; Houaiss, A.; Barbosa, F. A., eds.; Encyclopaedia Britannica do Brasil: RJ-SP, 1990.

5. Costa, P. R. R.; Pilli, R. A.; Pinheiro, S.; Vasconcelos, M. L. A. A.; Substâncias Carboniladas e seus Derivados, Bookman: Porto Alegre, 2003.

6. Livros texto de Química Orgânica Experimental trazem este experimento. Como exemplo, ver: Pavia, D. L.; Lampman, G. M.; Kriz, G. S.; Engel, R. G.; Introduction to Organic Laboratory Techniques, a Microscale Approach, $3^{\text {rd }}$ ed., Saunders College Publishing: Orlando, 1999; Palleros, D. R.; Experimental Organic Chemistry, Wiley: New York, 2000.

7. Merck Index; $12^{\text {th }}$ ed.; Budavari, S., ed.; Whitehouse Station: New Jersey, 1996.

8. Cânfora sintética foi adquirida por $\mathrm{R} \$ 74,00$ (setenta e quatro reais), pote de $500 \mathrm{~g}$ da marca Vetec.

9. Yoneda, J. D.; Leal, K. Z.; Seidl, P. R.; Azeredo, R. B. V.; Kleinpeter, E.; Quim. Nova 2007, 30, 2053.

10. Santos, A. P. B.; Gonçalves, I. R. C.; Pais, K. C.; Martinez, S. T.; Lachter, E. R.; Pinto, A. C.; Quim. Nova 2009, 32, 1667.

11. O éter etílico foi empregado devido à sua alta volatilidade, que permitiu a evaporação rápida e eficiente do solvente sobre uma placa de aquecimento em capela de exaustão. Em laboratórios de graduação que possuam evaporadores rotatórios, solventes menos voláteis e perigosos (acetato de etila, tetraidrofurano) podem e devem ser empregados.

12. van den Dool, H.; Kratz, P. H.; J. Chromatogr., A 1963, 11, 463.

13. Adams, R. P.; Identification of Essential Oil Components by Gas Chromatography/Mass Spectroscopy, Allured Publ. Corp.: Carol Stream, 2007. 
14. Wigfield, D. C.; Gowland, F. W.; Tetrahedron Lett. 1976, 17, 3373; Wigfield, D. C.; Gowland, F. W.; J. Org. Chem. 1977, 42, 1108; Wigfield, D. C.; Tetrahedron 1979, 35, 449.

15. Glass, R. S.; Deardorff, D. R.; Hebegar, K.; Tetrahedron Lett. 1980, 21, 2467; Yadav, V. K.; Jeyaraj, D. A.; Balamurugan, R.; Tetrahedron 2000, 56,7581 .

16. Suzuki, Y.; Kaneno, D.; Tomoda, S.; J. Phys. Chem. A 2009, 113, 2578.

17. Os livros foram consultados de acordo com a disponibilidade de versão em língua portuguesa e conhecimento de uso em disciplinas teóricas por conversa com pares. Eventualmente algum livro pode ter sido esquecido, ao que os autores antecipadamente pedem desculpas. Os livros pesquisados foram: McMurry, J.; Química Orgânica, Pioneira Thomson Learning: São Paulo, 2005; Vollhardt, K. P. C.; Schore, N. E.; Química Orgânica, Estrutura e Função, $4^{\mathrm{a}}$ ed., Bookman: Porto Alegre, 2004; Bruice, P. Y.; Química Orgânica, $4^{\mathrm{a}}$ ed., Pearson Prentice Hall: São Paulo, 2006; Solomons, T. W. G.; Fryhle, C. B.; Química Orgânica, $9^{a}$ ed., Livros Técnicos e Científicos: Rio de Janeiro, 2009; Morrison, R. T.; Boyd, R. N.; Química Orgânica, $8^{\mathrm{a}}$ ed., Fundação Calouste Gulbekian: Lisboa, 1983; Allinger, N. L.; Cava, M. P.; Jongh, D. C.; Johnson, C. R.; Lebel, N. A.; Stevens, C. L.; Química Orgânica, Guanabara Dois: Rio de Janeiro, 1976; Barbosa, L. C. A.; Introdução à Química Orgânica, Pearson Prentice Hall: São Paulo, 2004.

18. Um único livro texto consultado em português apresentou o papel do cátion na redução, tomando o $\mathrm{LiAlH}_{4}$ como exemplo: Constantino, M. G.; Química Orgânica, Curso Básico Universitário, LTC: Rio de Janeiro, 2008.

19. Clayden, J.; Greeves, N.; Warren, S.; Wothers, P.; Organic Chemistry, Oxford University Press: Florence, 2001; Carey, F. A.; Sundberg, R. J.; Advanced Organic Chemistry, Part B: Reactions and Synthesis, $4^{\text {th }}$ ed., Plenum Publishers: New York, 2001.

20. A diferença fundamental entre os mecanismos que mostram o papel coordenante do cátion $\mathrm{Na}^{+}$é o momento da solvólise (ou hidrólise): alguns autores mostram a transferência do próton pelo solvente no momento da transferência do hidreto, denotando a obrigatoriedade de uso de solvente prótico, enquanto outros mostram a possibilidade de protonação após a redução.
21. Bürgi, H. B.; Dunitz, J. D.; Shefter, E.; J. Am. Chem. Soc. 1973, 95 , 5065; Bürgi, H. B.; Lehn, J. M.; Wipff, G.; J. Am. Chem. Soc. 1974, 96, 1956; Bürgi, H. B.; Dunitz, J. D.; Lehn, J. M.; Wipff, G.; Tetrahedron 1974, 30, 1563.

22. Uma amostra de (-)-borneol $75 \%$ marca Fluka foi injetada no CG-DIC, e os $25 \%$ restantes da amostra foram inicialmente sugeridos como sendo isoborneol. Esta suposição foi confirmada com a igualdade nos dois tempos de retenção obtidos a partir da injeção do padrão àqueles dos produtos de redução da cânfora, só que com a inversão da área do sinal majoritário.

23. A análise da mistura não foi realizada por espectros de RMN por indisponibilidade de aparelho, e por esta razão todas as análises disponíveis na UFES foram realizadas como forma de compensar esta lacuna de interpretação. No caso de disponibilidade de realização dos espectros de RMN, ver Abraham, R. J.; Barlow, A. P.; Rowan, A. E.; Magn. Reson. Chem. 1989, 27, 1074.

24. Apesar de nossos resultados mostrarem a redução mais rápida em metanol (5 min de refluxo em erlenmeyer), há relatos na literatura de reduções mais rápidas em etanol: Serra, M. E. S.; Murtinho, D; de Melo, J. S.; Redução da Cânfora, exemplo de uma transformação selectiva, Boletim da Sociedade Portuguesa de Química (http://www.spq.pt/ boletim/docs/boletimSPQ_096_072_11.pdf), acessada em Outubro 2009.

25. Este resultado mostra a influência do solvente na diastereosseletividade da redução, e sugere uma participação do solvente na etapa de transferência do hidreto. No entanto, nenhuma discussão mais profunda foi realizada sobre este resultado experimental devido às dúvidas que ainda cercam este mecanismo. 